\title{
The Ross-Konno procedure for congenital aortic stenosis
}

\author{
Sameh M. Said^ \\ Division of Pediatric Cardiovascular Surgery, Masonic Children's Hospital, University of Minnesota, Minneapolis, MN, USA \\ Correspondence to: Sameh M. Said, MBBCh, MD, FACS. Associate Professor of Surgery and Pediatrics, Division of Pediatric Cardiovascular Surgery, \\ Masonic Children's Hospital, Department of Surgery, University of Minnesota, 2450 Riverside Ave S, AO Building, 521, Minneapolis, MN 55454, \\ USA. Email: ssaid@umn.edu.
}

\begin{abstract}
Left ventricular outflow tract (LVOT) obstruction can result from supravalvular, valvular and/or subvalvular etiologies. Congenital aortic valvular stenosis is frequently associated with aortic annular hypoplasia. Aortoventriculoplasty with pulmonary autograft, "The Ross-Konno" operation, provides more or less a radical solution to multilevel LVOT obstruction by enlarging the aortic annulus and the subvalvular area, thus relieving both valvular and subvalvular obstructions. In addition to this, the procedure carries the major advantage of having a competent autograft in the LVOT. An autograft that has the potential for growth and provides excellent quality of life without the need for anticoagulation. The procedure is most commonly performed as a complete root implantation, harvesting the coronary arteries as buttons, and harvesting the autograft with a muscle skirt to allow a single unit reconstruction of the LVOT. The procedure has been modified over time to minimize the risk of conduction tissue injury and the development of complete heart block by modifying the interventricular septal incision. The Ross-Konno procedure has changed the approach to patients with complex multilevel LVOT obstruction. However, while it can be performed early in life, one should be aware that this procedure is technically demanding and can be associated with a higher surgical risk. Nevertheless, it is considered "the ultimate solution" for those with complex multilevel LVOT obstruction.
\end{abstract}

Keywords: Ross; Ross-Konno; aortoventriculoplasty; autograft; hypoplastic aortic annulus

Submitted May 11, 2021. Accepted for publication Jul 19, 2021.

doi: 10.21037/acs-2021-rp-31

View this article at: https://dx.doi.org/10.21037/acs-2021-rp-31

\section{Introduction}

The Konno-Rastan operation, described contemporaneously by both Konno and colleagues (1) and Rastan and Koncz (2), has emerged initially as an alternative to apicoaortic conduit surgery for treatment of tunnel type subaortic stenosis. It significantly enlarges the left ventricular outflow tract (LVOT) and the aortic annulus by incising the interventricular septum (IVS), thus allowing for placement of an adequately sized aortic prosthesis (aortoventriculoplasty), and is considered as the procedure of choice for multilevel left ventricular outflow tact obstruction (LVOTO). The Ross procedure, first described in 1967 , utilizes the pulmonary autograft to replace the diseased aortic valve and became an attractive option in children and young adults due to the excellent quality of life, freedom from anticoagulation and more importantly, the maintenance of growth potential (3). The Ross procedure was initially applied to suitable cases without a significant size mismatch between the aortic and pulmonary roots. It was not until it was combined with the Konno aortoventriculoplasty (4) that it became an effective solution for young patients with complex LVOTO. The Ross-Konno operation is an excellent technique to completely eliminate

$\wedge$ ORCID: 0000-0003-2193-0314. 


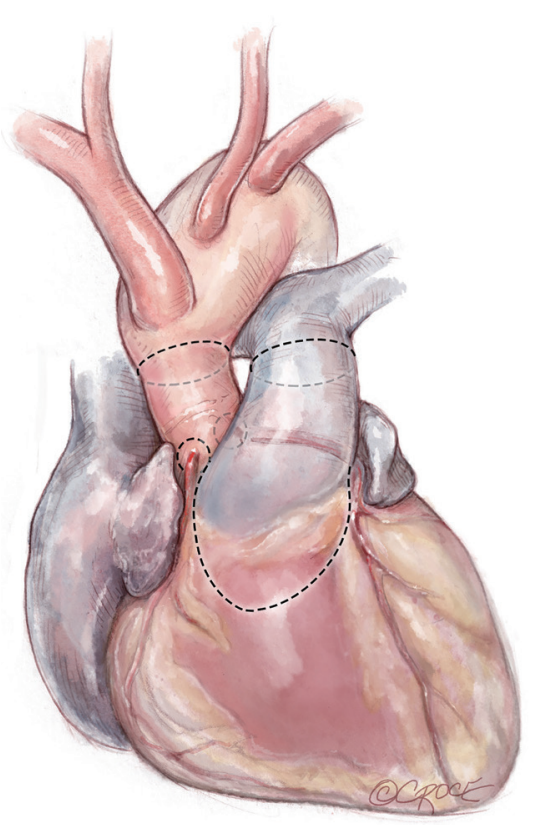

Figure 1 The proposed incisions for the pulmonary autograft harvest and ascending aorta transection. A skirt of right ventricular free wall muscle is harvested with the autograft for the planned anterior aortic root enlargement part (Konno) of the procedure.

multilevel LVOTO, albeit it is a technically demanding procedure.

\section{Operative techniques}

The most common implantation technique for the autograft is root replacement. This technique, when combined with the Konno procedure, allows its application in patients with size discrepancy between the native aortic annulus and the pulmonary autograft.

\section{Preparation}

After induction of general endotracheal anesthesia and placement of the necessary monitoring lines, including cerebral and somatic near-infrared spectroscopy (NIRS), the patient is positioned supine, prepped and draped as for standard median sternotomy. After sternotomy, a pericardial well is created and heparin is administered systemically. Distal ascending aortic/proximal arch cannulation is preferred to allow dissection and facilitate exposure. Both superior and inferior venae cavae are cannulated. Cardiopulmonary bypass is then initiated at

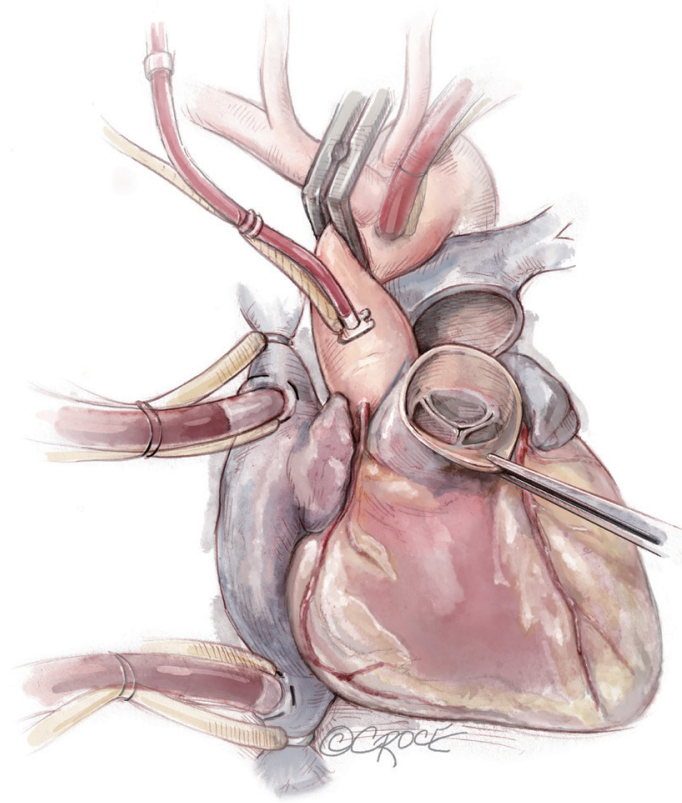

Figure 2 The distal ascending aorta and both cavae are cannulated. Once cardiopulmonary bypass is initiated, the main pulmonary artery is transected just proximal to its bifurcation and the pulmonary valve is inspected to determine its suitability as an autograft. This part can also be completed after arresting the heart and determining the non-suitability of the native aortic valve for repair.

mild hypothermia and a left ventricular vent is placed through the right superior pulmonary vein. The aorta and pulmonary artery are extensively mobilized, and the aortic root is dissected off the right ventricular outflow tract and the left atrial dome. Landmarks are identified for subsequent incisions (Figure 1). The pulmonary artery is transversely incised proximal to its bifurcation and the pulmonary valve is inspected to confirm its suitability as an autograft (Figure 2).

\section{Exposition}

The distal ascending aorta is cross clamped and antegrade cardioplegia is administered (we use del Nido cardioplegia). In the presence of significant aortic regurgitation, cardioplegia will be administered directly through the coronary ostia after transecting the aortic root. The ascending aorta is then transected and the aortic valve is inspected to determine whether repair is possible before the annulus is sized. In cases where the valve is not repairable, the aortic cusps are resected. 


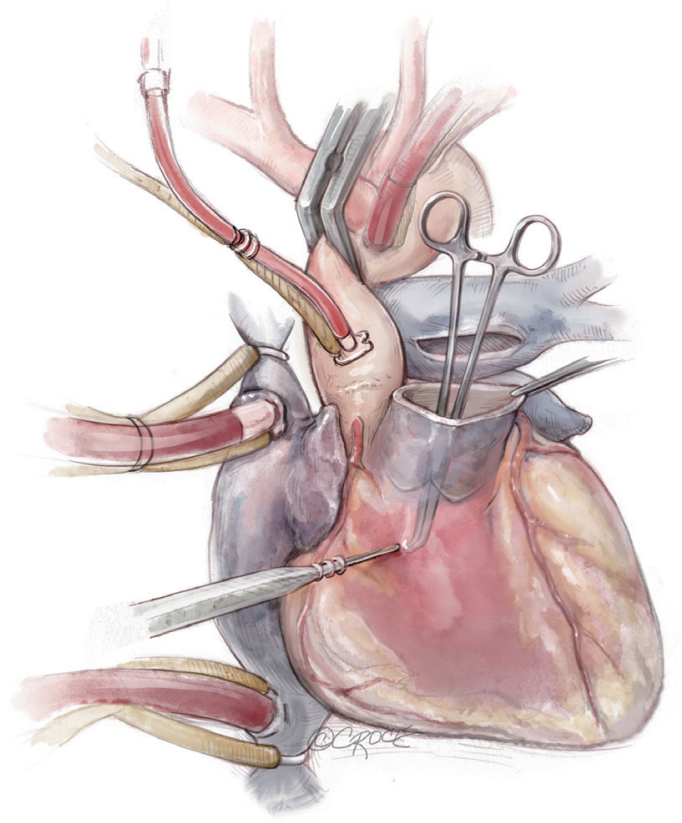

Figure 3 Once the pulmonary valve is determined to be suitable, the main pulmonary artery is completely transected and mobilized using electrocautery and the autograft is harvested. A right-angled clamp is passed through the pulmonary valve $5 \mathrm{~mm}$ below the nadir of the non-facing cusp and used as a guide to incise the right ventricular free wall safely below the pulmonary valve cusps.

\section{Operation}

\section{Pulmonary autograft assessment}

The final decision regarding the suitability of the pulmonary autograft for the Ross procedure is based on inspection the pulmonary valve after incising or transecting the main pulmonary artery. This includes the number and integrity of the leaflets, presence of any fenestrations and any significant degree of regurgitation. While intraoperative inspection is the key, some information can still be obtained from preoperative transthoracic echocardiography and crosssectional imaging studies or intraoperative transesophageal echocardiography, if available.

\section{Pulmonary autograft harvest}

If the pulmonary valve appears suitable, we proceed with harvesting of the autograft. We complete the transection of the main pulmonary artery proximal to its bifurcation and thoroughly mobilize the artery down to the level of the right ventricular muscle. A right-angled clamp is then passed through the pulmonary valve and is used as a guide

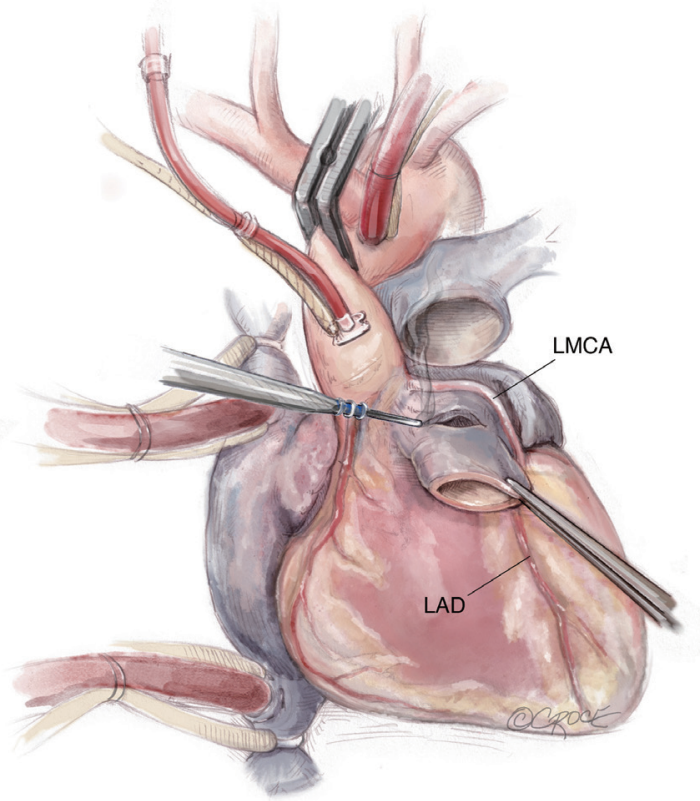

Figure 4 Lifting the pulmonary artery vertically from the plane of the heart during mobilization creates a gap from the coronary arteries. It is important to be aware of the close proximity of the left main and left anterior descending coronary arteries, especially in reoperative settings where landmarks are obscured. LMCA, left main coronary artery; LAD, left anterior descending.

to incise the right ventricular free wall below the pulmonary valve cusps (Figure 3).

The autograft is harvested (Figure 4) with a skirt of infundibular muscle that will be used to construct the Konno septal incision. The length of this skirt depends on the depth of the ventricular septal incision and the degree of enlargement required for the aortic root and LVOT. We use a combination of electrocautery and sharp dissection to harvest the autograft. The autograft is enucleated along its posterior attachment, paying attention to the course of the first septal perforator artery (Figure 5). If the plan is to use a prosthetic patch to reconstruct the ventricular septal incision, the infundibular muscle harvest is limited.

We administer cardioplegia to visualize all bleeding points from the autograft harvest site and either sutures or electrocautery is used to achieve hemostasis. It is important to ensure complete hemostasis, as this area is difficult to reach at the end of the procedure. At this stage, the autograft is sized at its base with Hegar or valve sizers and is placed in cold saline until its use. 


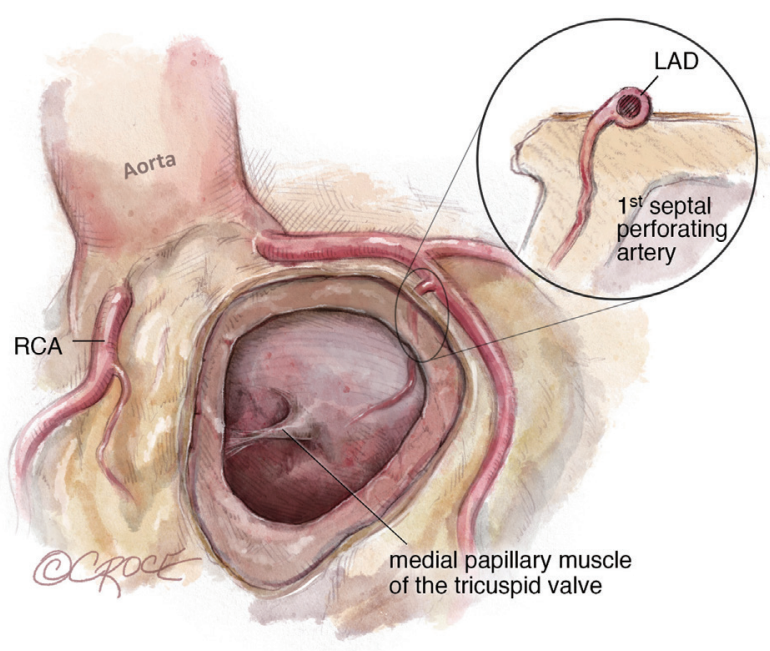

Figure 5 The autograft is harvested by a combination of sharp and electrocautery dissection. The autograft is carefully removed from its posterior attachment through "enucleation", thus preserving the first septal perforator branch of the left anterior descending coronary artery. A useful landmark for the location of the first septal perforator is the medial papillary muscle of the tricuspid valve. Administration of cardioplegia can help hemostasis at this stage by visualizing any bleeding point from the autograft harvest site. RCA, right coronary artery; LAD, left anterior descending.

\section{Coronary buttons harvest and preparation of the aortic root} The coronary buttons are then harvested and mobilized off the epicardial surface of the heart (Figure 6). Three to four milimeters of the native aortic root is kept in place, in addition to the non-coronary sinus, to support the autograft.

\section{Konno septal incision}

The Konno incision is typically placed to the left of the right coronary artery or between the commissural attachments of the right/left coronary sinuses across the ventricular septum. The extent of this incision depends on the degree of the size mismatch between the pulmonary autograft and the native aortic annulus and should also extend beyond the area of the subaortic stenosis. The Konno incision should be made in an oblique or transverse fashion and to the left of the medial papillary muscle of the tricuspid valve to avoid injury to the conduction tissue (Figure 7). Any associated septal myectomy or resection of endocardial fibroelastosis (EFE) is performed at this stage. A prosthetic patch (not preferred) may be used to construct

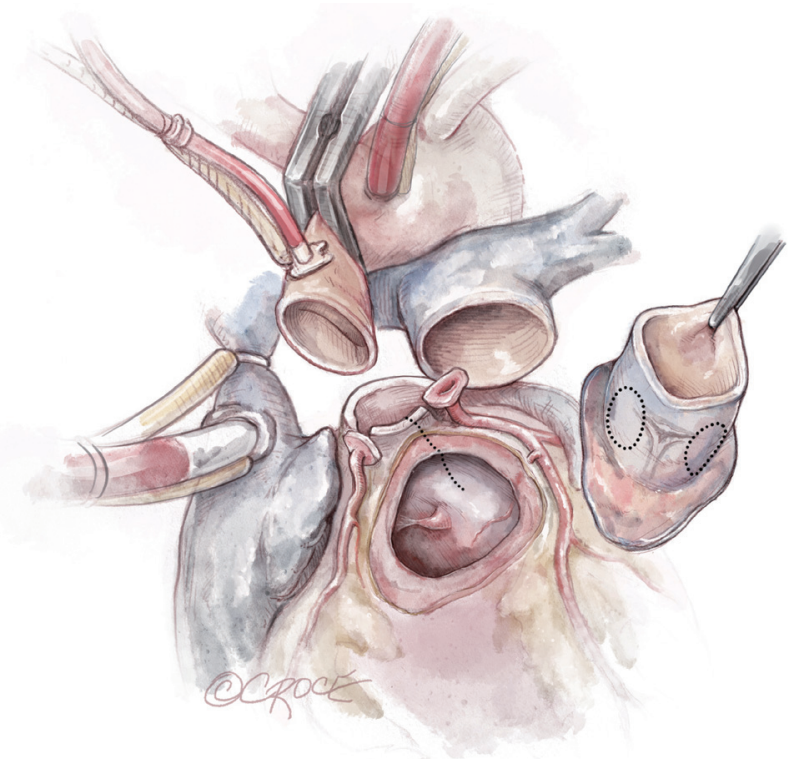

Figure 6 Preparation of the aortic root can be done prior to harvesting the autograft. The aortic valve leaflets are excised and the coronary buttons are harvested and mobilized off the epicardial surface of the heart. The left ventricular outflow tract is evaluated and a septal myectomy, and/or resection of endocardial fibroelastosis can be performed at this stage. Notice the proposed interventricular septal incision (Konno incision) (dotted line).

the septal incision if the extent of the incision is too long for the autograft to fill (Figure 8).

\section{Autograft implantation}

The autograft muscle should be trimmed, except in the area that will be used for the Konno septal incision reconstruction, in order to avoid the potential for subaortic obstruction from its bulk. We have used both running and interrupted sutures to implant the autograft depending on the nature and quality of the tissues. It is, however, critical to maintain symmetry of the autograft during reimplantation. We suture the remnants of the native aortic root to the implanted autograft using a running prolene suture as a hemostatic layer. A pericardial strip can also be used to support the autograft suture line.

\section{Coronary button reimplantation}

If the autograft is implanted correctly, the coronary button implantation sites occur most commonly in the middle of the facing sinus of Valsalva. Suitable openings 


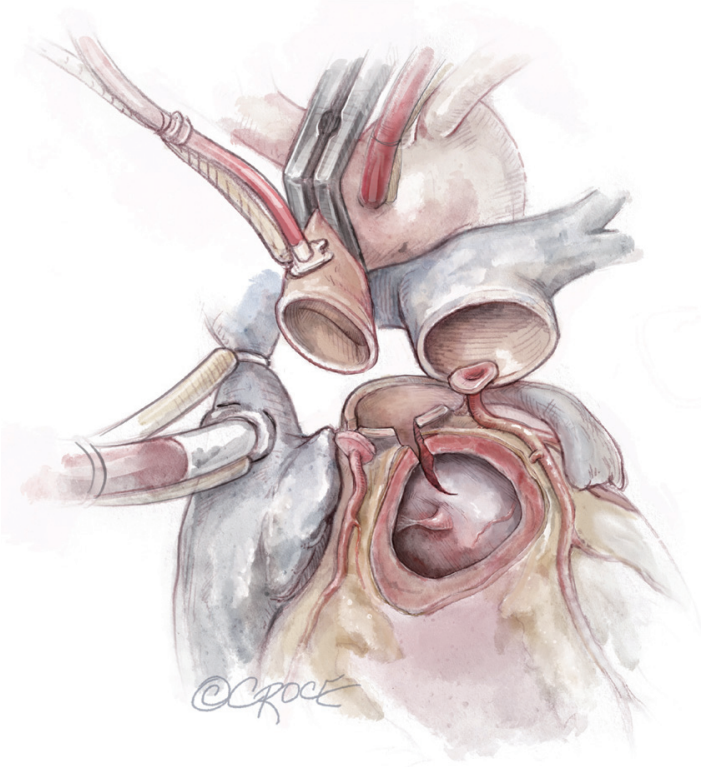

Figure 7 A Konno incision is performed to the left of the right coronary artery button or along the commissure between the left and right coronary sinuses of Valsalva. The extent of this incision depends on the degree of enlargement of the left ventricular outflow tract required and it should be done in an oblique or transverse fashion to avoid the conduction tissue.

in the autograft are created and the coronary buttons are implanted with a running prolene suture (Figure 9). Test dose cardioplegia can be administered to check for hemostasis at this point.

\section{Completion of the aortic reconstruction}

The distal autograft-aortic suture line is now completed (Figure 9). Cardioplegia can be administered to check for any potential bleeding points from the root at this stage, prior to removal of the aortic cross clamp.

\section{Pulmonary homograft implantation}

We routinely use the largest possible pulmonary homograft for reconstruction of the right ventricular outflow tract. The distal homograft anastomosis to the pulmonary artery bifurcation is performed using a running prolene suture (Figure 10), followed by completion of the anastomosis between the homograft and the right ventricular outflow tract, which we frequently perform on a strip of bovine pericardium to support the suture line (Figure 11).

\section{Completion}

The heart is then deaired and the aortic cross clamp is removed. Rewarming to normothermia is started with adequate inotropic support. Once at normothermia, the patient is ventilated and weaned off cardiopulmonary bypass and transesophageal echocardiography is performed to assess both the right and LVOTs, the neoaortic valve and the pulmonary homograft, as well as ventricular function. The rest of the procedure is completed as routine.

\section{Pitfalls}

\section{Coronary arteries}

Paying attention to the course of the left main coronary artery and left anterior descending (LAD) artery is critical during the autograft harvest, especially in reoperative settings where visualization can be easily impaired. We use a coronary probe to help localize the epicardial coronary arteries in this circumstance. The pulmonary autograft is enucleated carefully along its posterior attachment to avoid injury to the first septal perforator branch of the LAD.

Stay sutures placed in the middle portion of each coronary button facilitates harvesting and maintains orientation of the coronary buttons to prevent kinking during reimplantation.

Prior to implantation of the right coronary button, the distal aortic to autograft reconstruction can be initiated to fix the autograft in position and prevent its rotation, thus avoiding any potential kink/twist in the right coronary artery button when implanted. In patients with concomitant aortic arch hypoplasia, it is not uncommon for the pulmonary bifurcation to be located posterior to the neoaorta. To minimize the potential risk of coronary compression secondary to the homograft, translocation of the distal homograft anastomosis to the left pulmonary artery may be needed.

\section{Small native aortic annulus/autograft-to-native aortic annulus size mismatch}

In the presence of a small size mismatch between the pulmonary autograft and the native aortic annulus, other aortic root enlargement techniques can be considered, such as posterior Nicks' procedure or Manouguian's procedure to avoid the risks associated with the Konno incision and to sufficiently open the root and accommodate the autograft. 
A

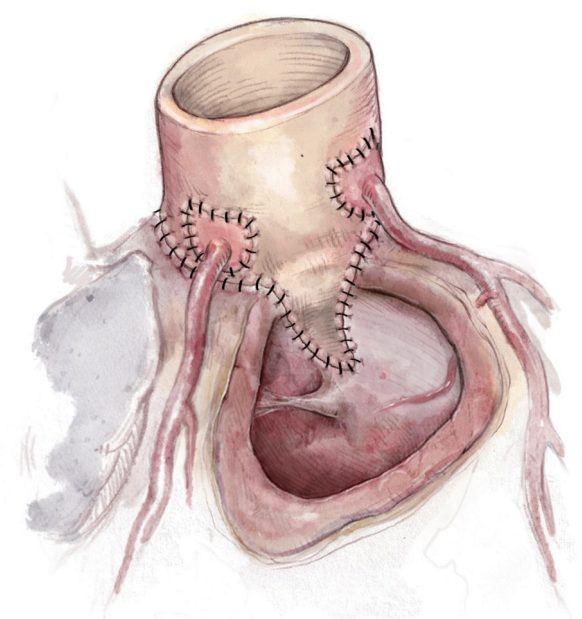

B

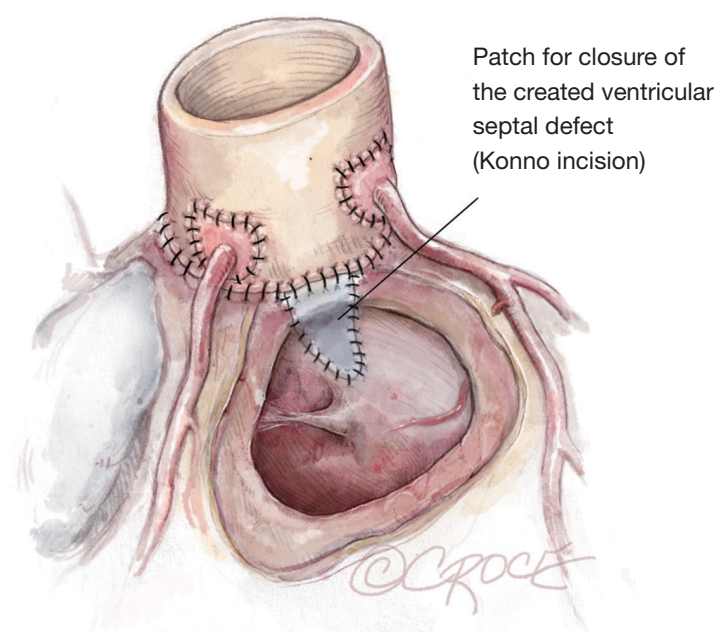

Figure 8 The pulmonary autograft with its infundibular extension is then implanted in the left ventricular outflow tract (A). This can be performed using running or interrupted sutures. It is important to trim the infundibular skirt to fit the septal incision and minimize the risk of outflow tract obstruction by leaving a bulk of infundibular muscle below the neoaortic valve. (B) An alternative option, especially when the length of the septal incision is longer than the infundibular skirt, is to close the created ventricular septal defect with a prosthetic patch and reimplant the autograft as a full root technique.

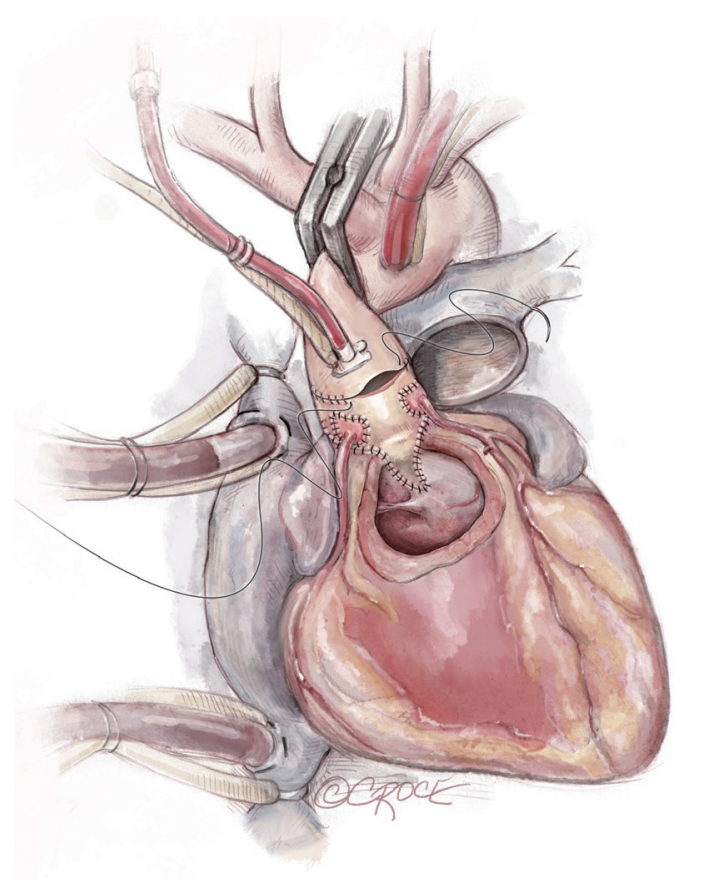

Figure 9 The left followed by the right coronary arteries are implanted in the middle of the corresponding sinuses of Valsalva of the autograft. The completed autograft and the reconstructed neoaortic root are shown. The distal pulmonary homograft anastomosis can be done at this stage prior to reconstructing the ascending aorta to facilitate exposure and ensure hemostasis.

\section{Konno septal incision}

It is important to maintain the orientation of the Konno septal incision in an oblique or transverse fashion to avoid injury to the conduction tissue.

\section{Autograft implantation}

Maintaining symmetry of the autograft during reimplantation is critical. It is important to stabilize the distal autograft/ native aorta suture line using a strip of prosthetic material especially in older patients to prevent autograft dilatation and minimize the risk of future neoaortic valve regurgitation. If a running suture technique is used for autograft implantation, it is important to maintain proper orientation and avoid distortion of the autograft and/or injury to the autograft valve.

\section{Concomitant lesions [ventricular septal defect (VSD)/aortic arch hypoplasia]}

In the presence of associated VSD, it is preferred not to use the infundibular muscle to close the defect and to use a prosthetic patch. This minimizes the risk of inflow obstruction to the pulmonary valve resulting from suturing the bulky infundibular muscle to the tricuspid valve annulus.

\section{Pulmonary homograft implantation}

The pulmonary homograft distal anastomosis in some 


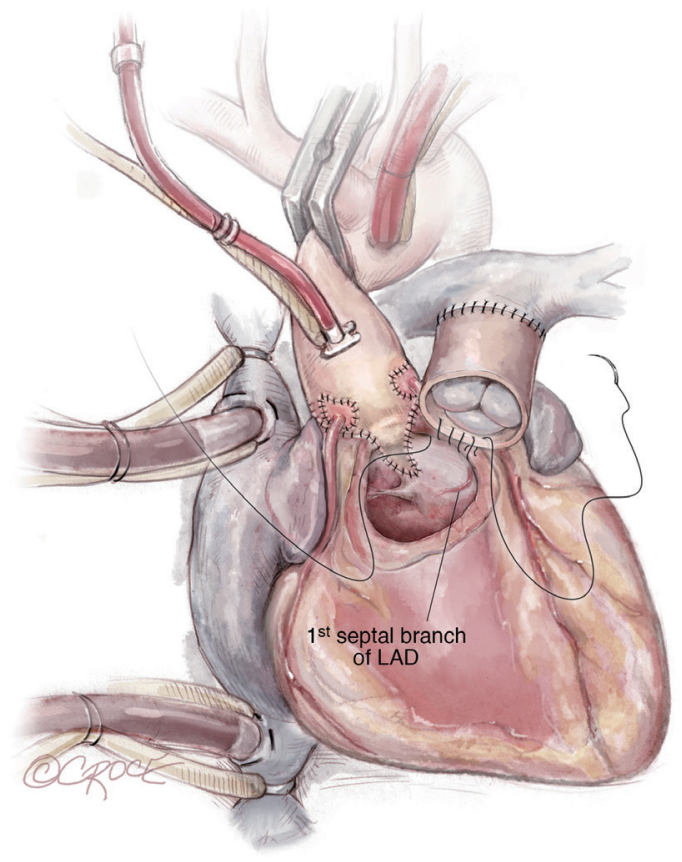

Figure 10 Implantation of the pulmonary homograft. The distal anastomosis is constructed to the pulmonary artery bifurcation, followed by suturing the proximal homograft to the right ventricular outflow tract. It is important to take the endocardium and part of the myocardium only along the posterior suture line to avoid compromising the first septal perforator branch of the left anterior descending coronary artery which is in close proximity. This can be supported with a strip of pericardium to ensure hemostasis. We prefer to use the largest possible pulmonary homograft in this position.

patients is preferred to be performed prior to completion of the distal autograft/aortic anastomosis to facilitate exposure and ensure hemostasis. The proximal homograft to right ventricular outflow tract suture line is taken through the endocardium and partial thickness of the myocardium to avoid compromising the septal perforator branch of the LAD. While pulmonary homograft implantation can be performed on beating heart. We should exercise caution in making this decision to avoid tearing of the right ventricular muscle or the homograft during implantation.

\section{Modified Ross-Konno procedure}

\section{The mini Ross-Konno}

A modification of the Ross-Konno procedure without creation of a VSD is known as a "mini Ross-Konno" (5).

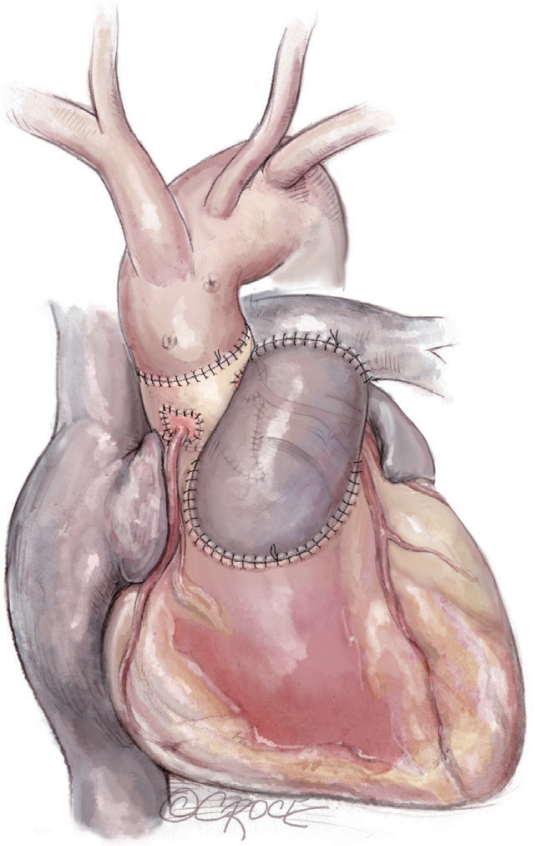

Figure 11 The completed Ross-Konno operation.

The initial steps of the procedure are similar to the standard Ross-Konno. After harvesting the autograft, excising the aortic valve and harvesting the coronary buttons, an extended left ventricular septal myectomy is performed, instead of a VSD, to eliminate all potential areas of subvalvular obstruction. The aortic annulus is then enlarged to accommodate the autograft. The rest of the procedure is completed as previously described (Figure 12). This modification provides adequate relief of the LVOTO and at the same time, minimizes some of the risks associated with the standard Konno incision.

\section{The subcoronary Ross-Konno}

The original description of the implantation technique of the pulmonary autograft followed by a subcoronary technique of implantation in which the coronary buttons are not excised. The advantage of this technique is that the autograft is completely supported by the native aortic root. This technique was popularized by Murin and colleagues for the Ross-Konno procedure (6). The Konno incision is created, and a patch is used to reconstruct the IVS. An additional patch to augment the non-coronary sinus is required. The autograft is then implanted beneath the coronary ostia and the remainder of the procedure is conducted as previously described for the standard Ross- 


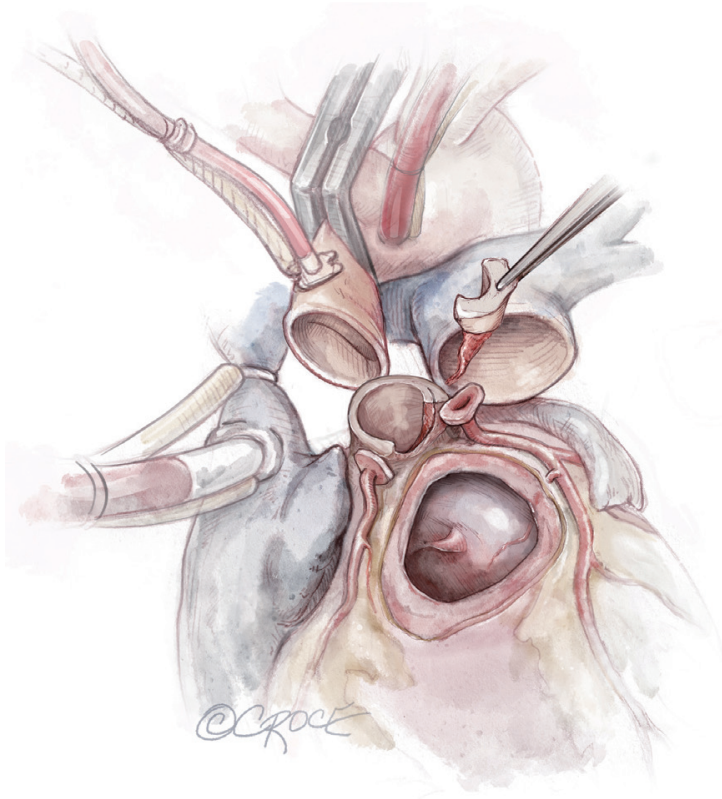

Figure 12 The mini Ross-Konno procedure. The initial steps of the procedure are similar to the standard Ross-Konno. However, a smaller Konno incision combined with an extended left ventricular septal myectomy is performed to eliminate all potential subaortic obstruction without the creation of a ventricular septal defect. The aortic annulus is then enlarged to accommodate the autograft.

\section{Konno.}

Supporting the autograft completely with the native aortic root and LVOT may result in higher freedom from reoperation due to autograft dilatation in comparison to standard root technique and avoids the risks related to coronary reimplantation. The major disadvantages are the length of the Konno incision required to accommodate the autograft and the use of prosthetic materials in the aortic root.

\section{Modification of the ventriculoplasty incision}

The modified Ross-Konno was introduced in 2005 with the goal of placing the incision for LVOT enlargement away from the IVS, thus decreasing the risk for conduction tissue injury (7) (Figure 13). The LVOT is enlarged by incising the annulus and the left ventricular free wall posterior to the course of the left main coronary button. This requires mobilization and dissection of the left main coronary artery button similar to an arterial switch operation. The autograft is then implanted in the LVOT using multiple interrupted pledgeted mattress sutures which are necessary to maintain autograft stability and provide adequate hemostasis (Figure 14).

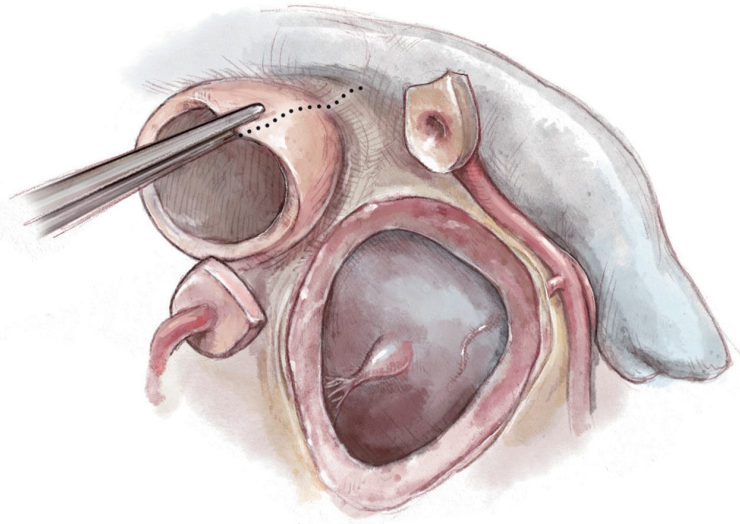

Figure 13 The modified Ross-Konno procedure is performed by amending the ventricular septal incision. The left ventricular outflow tract is enlarged posterior to the course of the left main coronary artery (dotted line), thus avoiding the interventricular septum and the conduction tissue.

\section{Comments}

\section{Clinical results}

The estimated risk of early mortality has been close to $10 \%$; however, this decreases to less than $5 \%$ in experienced hands. The majority of early morbidity is related to dysrhythmias and conduction abnormalities. Ventricular tachycardia has been reported in up to $28 \%$ in the postoperative period (8), while the incidence of heart block ranged from $0-6 \%$ (9). Freedom from reoperation due to recurrent LVOTO has been satisfactory with estimated 5and 10 -year freedom of over $95 \%$ and $90 \%$ respectively (10). Reoperation is mostly related to new progressive aortic valve regurgitation.

\section{Advantages of this technique}

In addition to the inherent advantages related to having an autograft in the aortic position (excellent hemodynamics, growth potential, lack of need for anticoagulation and lower incidence of infection), several other advantages are present. In experienced hands, the procedure can be performed in neonates and infants with excellent results, which may minimize and/or avoid repeat interventions and provide a longer-term relief of LVOTO. the procedure can also maintain good ventricular function in comparison to other palliative options, such as open or balloon valvotomy. Using the harvested infundibular muscle skirt to patch the ventricular septal incision optimizes the neo-aortic root and 


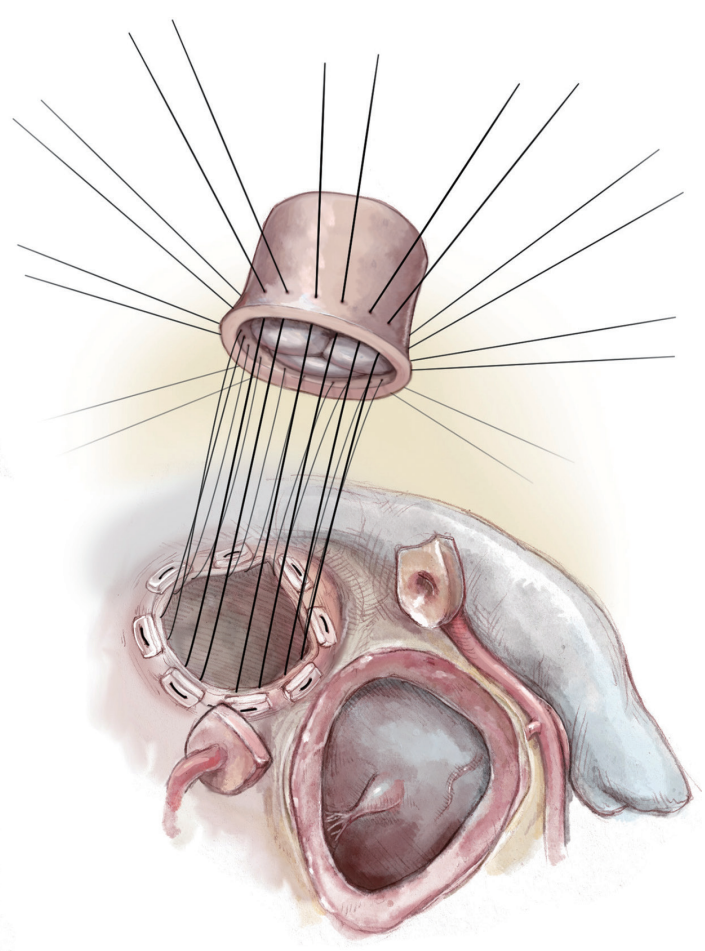

Figure 14 The left ventricular outflow tract is enlarged posteriorly by incising the free wall of the left ventricle posterior to the course of the left main coronary artery. The autograft is then implanted using multiple pledgeted mattress sutures to ensure stability and hemostasis.

LVOT geometry, which allows regular and proportional growth. Moreover, the procedure can be combined with the resection of EFE in neonates with hypoplastic aortic annulus and critical aortic stenosis, which may result in the avoidance of a single ventricle pathway. Lastly, the procedure can also be extended to patients with Shone's complex or an interrupted aortic arch, whether as a primary or as a secondary procedure after initial palliation.

\section{Caveats}

\section{Sub-coronary Ross-Konno}

While the Ross-Konno procedure is commonly performed as a root replacement, Murin and colleagues reported the use of the subcoronary technique for autograft implantation in 13 children with severe LVOTO (6). The results were good with an early mortality of $7.7 \%$ (one patient) and a median follow-up of 20 months with no mortality and no interventions on the outflow tract or the aortic valve. It is important, however, to realize that the success of this technique relies on accurate and symmetrical implantation of the autograft in the aortic root to avoid neoaortic valve regurgitation. It is also important to recognize that the Konno incision disrupts the aortic annulus and using any patches to reconstruct the root will interfere with root symmetry and the geometry of the LVOT. Most of the Ross-Konno patients are neonates and infants and it will be quite challenging to perform a subcoronary implantation in these patients due to the small roots (11).

\section{Use of prosthetic patches to reconstruct the ventriculoplasty incision}

While it is possible to reconstruct the Konno incision with a prosthetic patch, it is not preferred, especially in neonates and infants as this increases the complexity of construction and may result in increased rigidity and subsequent immobilization of the anterior portion of the neoaortic root. Using the infundibular muscle skirt that is harvested with the autograft for constructing the ventricular septal incision allows for more uniform geometry of the LVOT and preserves the potential for growth.

\section{Disadvantages of Ross-Konno procedure}

While it may seem that the Ross-Konno procedure places two valves at risk for what starts as a single valve disease, the limited options for aortic valve replacement and the lack of appropriately sized prostheses in children, especially neonates and infants, make this procedure a good option to consider.

There may also be concern related to using an infundibular muscle skirt. While using this tissue to reconstruct the Konno septal incision maintains geometry and growth potential in the LVOT, complications related to residual VSDs, false aneurysms and right ventricular outflow tract obstruction may occur in some patients (12).

\section{Long-term performance of the autograft and homograft}

Reoperation for pulmonary conduit failure is more frequent in young children. While this may be due in part to younger age at operation, this could also be related to the choice of the conduit. Factors that adversely affect the longevity of the homografts in the pulmonary position include the use of aortic homografts rather than pulmonary homografts and smaller homograft size. It is advisable to use an oversized pulmonary homograft whenever possible at the time of the procedure. A common indication for reoperation is related 
to progressive neoaortic valve regurgitation, which is mostly related to annular or sinotubular junction dilatation. Experience from the Ross procedure shows excellent results with the autograft and freedom from reoperation due to neoaortic valve regurgitation that surpasses $85-90 \%$ in the mid-term. It remains to be determined if the Ross-Konno operation will have similar autograft outcomes and whether these outcomes will be different between infants and older patients.

\section{Ventricular function, interactions and electromechanical dyssynchrony}

Long-term follow-up after Ross-Konno shows a chronic increase in afterload and a decrease in global left ventricular longitudinal strain. This occurs independently to autograft function and has been demonstrated by the study performed by Schäfer and colleagues (13). The authors reviewed 13 patients who underwent the Ross-Konno operation and were evaluated with cardiac magnetic resonance imaging. They also demonstrated impairment in left ventricular intraventricular mechanics and electrical dyssynchrony.

The effect of this complex procedure on late left ventricular function has been a concern. This could be related in part to the interventricular septal incision and/ or the changes in left ventricular geometry. In a study by Hoashi and colleagues that included 14 patients younger than 20 years of age, the authors demonstrated that left ventricular contraction and volume normalize long after the operation. However, ventricular compliance remains reduced despite adequate relief of LVOTO (14).

In conclusion, the Ross-Konno operation is considered the ultimate solution for complex and multilevel LVOTO. It offers several advantages over mechanical prostheses and homograft replacements of the aortic valve/root, especially in young patients in whom quality of life, freedom from lifelong anticoagulation and the potential for ongoing growth are critical. The procedure, however, is technically demanding and requires expertise to achieve excellent outcomes.

\section{Acknowledgments}

Funding: None.

\section{Footnote}

Conflicts of Interest: SMS is a consultant for Cryolife, and Stryker.
Open Access Statement: This is an Open Access article distributed in accordance with the Creative Commons Attribution-NonCommercial-NoDerivs 4.0 International License (CC BY-NC-ND 4.0), which permits the noncommercial replication and distribution of the article with the strict proviso that no changes or edits are made and the original work is properly cited (including links to both the formal publication through the relevant DOI and the license). See: https://creativecommons.org/licenses/by-nc-nd/4.0/.

\section{References}

1. Konno S, Imai Y, Iida Y, et al. A new method for prosthetic valve replacement in congenital aortic stenosis associated with hypoplasia of the aortic valve ring. J Thorac Cardiovasc Surg 1975;70:909-17.

2. Rastan H, Koncz J. Aortoventriculoplasty: a new technique for the treatment of left ventricular outflow tract obstruction. J Thorac Cardiovasc Surg 1976;71:920-7.

3. Ross DN. Replacement of aortic and mitral valves with a pulmonary autograft. Lancet 1967;2:956-8.

4. Reddy VM, Rajasinghe HA, Teitel DF, et al. Aortoventriculoplasty with the pulmonary autograft: the "Ross-Konno" procedure. J Thorac Cardiovasc Surg 1996;111:158-65; discussion 165-7.

5. Al Halees Z. The Mini-Ross-Konno procedure. Eur J Cardiothorac Surg 2011;39:1067-9.

6. Murin P, Sinzobahamvya N, Schulz A, et al. Modified Ross-Konno procedure in children: subcoronary implantation technique with Konno incision for annular and subannular hypoplasia. Interact Cardiovasc Thorac Surg 2018;27:264-8.

7. Mavroudis C, Backer CL. Technical tips for three congenital heart operations: modified Ross-Konno procedure, optimal ventricular septal defect exposure by tricuspid valve incision, coronary unroofing and endarterectomy for anomalous aortic origin of the coronary artery. Oper Tech Thorac Cardiovasc Surg 2010;15:18-40.

8. Hraska V, Krajci M, Haun Ch, et al. Ross and Ross-Konno procedure in children and adolescents: mid-term results. Eur J Cardiothorac Surg 2004;25:742-7.

9. Hraska V, Lilje C, Kantorová A, et al. Ross-konno procedure in children: midterm results. World $\mathrm{J}$ Pediatr Congenit Heart Surg 2010;1:28-33.

10. Matsuzaki Y, Hiramatsu T, Agematsu K, et al. Long-term outcomes of Ross and Ross-Konno operations in patients under 15 years of age. Gen Thorac Cardiovasc Surg 
2019;67:420-6.

11. Barron DJ. The sub-coronary Ross-Konno procedure in children: a word of caution for use in infants. Interact Cardiovasc Thorac Surg 2018;27:314-5.

12. Mavroudis C, Mavroudis CD, Jacobs JP. The Ross, Konno, and Ross-Konno operations for congenital left ventricular outflow tract abnormalities. Cardiol Young 2014;24:1121-33.

Cite this article as: Said SM. The Ross-Konno procedure for congenital aortic stenosis. Ann Cardiothorac Surg 2021;10(4):527-537. doi: 10.21037/acs-2021-rp-31
13. Schäfer M, Browne LP, von Alvensleben JC, et al. Ventricular interactions and electromechanical dyssynchrony after Ross and Ross-Konno operations. J Thorac Cardiovasc Surg 2019;158:509-17.

14. Hoashi T, Kagisaki K, Kurosaki K, et al. Late left ventricular function after successful Ross-Konno operation. Ann Thorac Surg 2013;96:196-201. 